\title{
Optimal Control Theory for the Design of Optical Waveguides
}

\author{
D. K. Pant, Rob D. Coalson, Marta I. Hernández, and José Campos-Martínez
}

\begin{abstract}
Techniques of optimal control theory, previously developed to assist in the design of ultrafast laser pulses for controlling laser-molecule interactions, are adapted to aid in the design of optical waveguides that can be modeled via the paraxial equation. Noting that the paraxial equation is isomorphic to the time-dependent Schrödinger Equation, previous work focussing on control of quantum systems can be directly applied to the problem of waveguide design. Specific application is given to the design of S-bend waveguides. It is shown how optimal control theory yields an algorithm which can refine an initial guess for the index of refraction profile in order to minimize a cost function which reflects design goals. Numerical examples are presented to illustrate the utility and flexibility of the proposed technique.
\end{abstract}

Index Terms - Calculus of variations, coherent control, Lagrange multipliers, Maxwell's Equations, optimal control theory, paraxial equation, S-bend waveguide, steepest descents.

\section{INTRODUCTION}

$\mathbf{O}$ PTICAL waveguides play an important role in communications technology and as logical elements in optically based computing devices [1]. From the point of view of theory, a major challenge is to predict an index of refraction profile that will guide input light in the desired way, subject to constraints that enable practical fabrication of the waveguide.

The fundamental theoretical framework for understanding the propagation of light through materials is Maxwell's Equations [2], a set of coupled vectorial time-evolution equations for the three dimensional electric and magnetic field distributions. While straightforward to solve in principle [3], considerable computer time and memory is required, especially in the simulation of waveguide propagation, which requires that accurate long-time steady state behavior be numerically monitored [4].

Fortunately, light propagation in optical waveguides fabricated from semiconductor materials such as GaAs and AlGaAs [5] is generally well-described by the approximation of scalar optics, namely Helmholtz' Equation [6], [7]. In fact, an approximate version of Helmholtz' Equation known as the paraxial equation is usually adequate [1], [8]. The paraxial equation prescribes unique evolution along the propagation direction of a component of the electric or magnetic field,

Manuscript received June 6, 1997; revised October 3, 1997. This work was supported by NATO under Grant CRG 941284. The work of R. D. Coalson was supported in part by the NSF under Grant CHE-9529674.

D. K. Pant and R. D. Coalson are with the Department of Chemistry, University of Pittsburgh, Pittsburgh, PA 15260 USA.

M. I. Hernández and J. Campos-Martínez are with the Instituto de Matemáticas y Física Fundamental (CSIC), Serrano 123, E-28006 Madrid, Spain.

Publisher Item Identifier S 0733-8724(98)00637-9. given its value at the point of injection into the waveguide. Standard numerical algorithms such as the beam propagation method (BPM) [9], [10] can reliably generate the selected component of the electromagnetic field everywhere in space once the index of refraction profile is specified.

While it is very useful to have methods for computing electromagnetic fields associated with a specific index profile, their availability does not in itself provide a direct way to optimize the index profile in order to produce a desired guided light beam. One can propose an index profile (on intuitive grounds) and then compute the associated field component. The output field distribution hopefully suggests a way to modify the index profile in order to improve the desired guiding, but this often amounts to an inefficient trial and error strategy.

The paraxial equation of scalar optics is isomorphic to the time-dependent Schrödinger Equation of quantum mechanics in two space plus one time degree of freedom. That is, the time variable in quantum mechanics maps onto the propagation coordinate (say, $z$ ) of the optical waveguide. The two transverse coordinates $x, y$ in the waveguide problem map onto spatial degrees of freedom in quantum mechanics. The index of refraction profile in optics maps onto the potential energy function in quantum mechanics (details are given below). The dilemma outlined in the preceding paragraph is also encountered in modern molecular quantum mechanics. Namely, given a potential energy function it is possible (at least for small molecules) to solve the time-dependent Schrödinger Equation in order to predict how an initially prepared state will evolve in time, but one would often like to know what potential function generates a measured output state, or even more ambitiously, what potential function will generate a desired output state at some particular time.

These concerns have become of great interest in the molecular quantum mechanics community with the advent of sophisticated ultrafast (subpicosecond) laser instruments and detection schemes [12]. It is now possible, in prototypical cases, to "sculpt" the potential function, in particular the coupling of the molecular dipole to the applied time-dependent laser field, to guide a molecular wavepacket through space and time [13]. The desire to assist with such "coherent control" experiments has spurred the development of "optimal control" algorithms [14] that enable systematic and intelligent modification of the potential function (more precisely, the applied electric field pulse) to achieve desired wavepacket propagation.

The same strategy can be applied to the design of optical waveguides. This follows immediately from 1) the existence 
of well-defined methodologies for optimizing molecular potentials in order to achieve desired wavepacket propagation according to the time-dependent Schrödinger Equation, and 2) the isomorphism between Schrödinger and paraxial equations noted above. In this paper we explain the procedure by considering a simple yet nontrivial problem in optical waveguide design, namely how to construct an S-bend guide that takes a light beam incident at one point along the transverse $x y$-plane and guides it to another point along the plane over a fixed propagation distance. The S-bend guide shape is represented as a Fourier series, and equations/algorithms for optimizing the Fourier coefficients are provided and illustrated by numerical example. The procedure can handle an arbitrary number of coefficients (CPU time scales roughly linearly with the number of coefficients) and can easily be generalized to more complex waveguide structures.

We also illustrate the ability of the optimal control procedure to incorporate a range of costs or penalties which may figure into the design strategy. In the present illustration, we consider the desire to achieve the "bend" in the S-guide as rapidly as possible along the propagation direction, in order to make the device as compact as possible. We devise an appropriate cost function that favors rapid bending. Beyond a certain point, rapid bending can only be achieved at the cost of some degradation in the guiding accuracy - the designer decides what the most important objective is and builds this into the design prescription.

An outline of the paper is as follows. In Section II, we briefly review important details of the paraxial equation. In Section III, we adapt principles of Optimal Control Theory that have previously been applied to the time-dependent Schrödinger Equation [14] to the paraxial equation. In particular, we consider the problem of optimizing an S-guide to produce a desired output electric field at a prescribed distance from the entrance to the guide. In Section IV, we illustrate the procedure numerically using parameters relevant to the design of GaAs/AlGaAs optical waveguides [5]-[7]. Section V summarizes the work.

\section{THE PARAXIAL EQUATION}

For many waveguides the Helmholtz Equation [1], [2] provides a good description of the steady state field amplitudes. Namely, for a given index of refraction profile $n(\vec{x})$

$$
\left[\nabla^{2}+n^{2}(\vec{x}) k^{2}\right] E(\vec{x})=0
$$

where $\vec{x}=(x, y, z), \nabla^{2}$ is the three dimensional Laplacian operator, $E(\vec{x})$ is the appropriate component of the electric field, and $k=2 \pi / \lambda$ where $\lambda$ is the free space wavelength of the injected light. In standard optical waveguides the index profile is only weakly dependent on the propagation direction, $z$. In this case it is useful to write $E(\vec{x})=\exp \left(i \beta_{0} z\right) \psi(\vec{x})$, where $\beta_{0}$ is an appropriate "reference propagation constant" which factors out as much of the $z$ dependence in $E$ as possible. The standard synthesis of waveguides entails deposition of a thin layer of "impurity" material on a bulk substrate to form the guiding region. For devices fabricated in this manner a good choice for the reference index is $\beta_{0}=k n_{0}$, with $n_{0}$ the index of refraction of the substrate.

If the index difference between the bulk and the guiding regions of the waveguide is much less than the absolute value of the bulk index, electric fields guided by the device will be characterized by an amplitude factor $\psi$ that varies slowly with $z$. In this case, the evolution of $\psi$ is accurately described by a partial differential equation which is first order in $z$, namely

$$
i \frac{\partial \psi(x, y, z)}{\partial z}=\left[\frac{-1}{2 \beta_{0}} \nabla_{T}^{2}-k \Delta n(x, y, z)\right] \psi(x, y, z) \text {. }
$$

Here $\nabla_{T}^{2} \equiv \partial^{2} / \partial x^{2}+\partial^{2} / \partial y^{2}$ is the transverse Laplacian, and $\Delta n(x, y, z) \equiv n(x, y, z)-n_{0}$ is the deviation of the index of refraction from its bulk value at any point in space. The condition $\Delta n / n_{0}$ has also been invoked to neglect a term proportional to $\Delta n^{2}$ on the right hand-side (RHS) of (2).

Clearly, (2), known as the paraxial equation [1], [8], has the form of a time-dependent Schrödinger equation two "space" $(x, y)$ and one "time" coordinate $(z)$. The role of the effective potential in the equivalent Schrödinger equation is played essentially by $-\Delta n(x, y, z)$. The fact that light is trapped in regions of space with higher values of refractive index is consistent with the equivalent quantum mechanical scenario in which a wavepacket is bound in a potential well (region of low potential energy relative to its surroundings).

Of the two directions transverse to the propagation direction, the dependence of the electric field on the direction parallel to the "deposition" surface, say $x$, is important, since it determines whether light is effectively confined in this direction. The details of the electric field along the other transverse direction ( $y$ pointing into the substrate) are less important. The light is attenuated as one moves further into the bulk, but the details are not critical for most waveguide design issues. Fortunately, the dependence of the paraxial equation on coordinate $y$ can be eliminated to a good approximation using the effective index method (EIM) [1]. For each $z$ the EIM provides a procedure for obtaining the effective index along the important transverse direction $x$, to be denoted $n_{\mathrm{eff}}(x, z)$. The paraxial equation then simplifies to

$$
i \frac{\partial \psi(x, z)}{\partial z}=\left[\frac{-1}{2 \beta_{0}} \frac{\partial^{2}}{\partial x^{2}}-k \Delta n(x, z)\right] \psi(x, z)
$$

with $\Delta n(x, z) \equiv n_{\mathrm{eff}}(x, z)-n_{0}$.

In this paper we shall concern ourselves with the paraxial equation in one "confinement" direction $(x)$ and one propagation direction $(z)$, assuming that for a given index profile $\Delta n(x, z)$ the solution of this equation accurately represents the distribution of the electric field in the waveguide under steady-state conditions. The goal is to select the index profile that generates the best guiding results. Suppose, for example, that we inject a spot of light at the beginning of the waveguide $(z=0)$ at the lateral position $x=x_{0}$. At the output of the waveguide a distance $L$ down the propagation axis, we want the spot to be located at lateral position $x=x_{f}$. What index of refraction function $\Delta n(x, z)$ generates an output state which best matches the appropriate target state (e.g., the fundamental mode of the waveguide into which the light is transferred at $z=L)$ ? 
Since there are many possible motifs for the construction of the waveguide, let us be more specific. We shall consider a class of profiles for which at any point along the propagation distance $z$ the waveguide is centered at $x_{c}(z)$ but the shape of the waveguide remains the same. For simplicity, we consider a piecewise continuous profile with the guiding region defined by a strip of width $2 d$ (centered at $x_{c}(z)$ ). The (effective) index inside the guide is $n_{\text {guide }}$ and outside the guide it is $n_{0}$ such that $n_{\text {guide }}>n_{0}$. Thus, the problem is to pick the guiding curve $x_{c}(z)$ which optimizes the output field for given input conditions. This is a problem which can be solved in a systematic and efficient manner using optimal control theory.

\section{OPTIMAL CONTROL THEORY}

To simplify notation, and to connect easily to the quantum mechanical literature upon which our treatment is based, note that the paraxial equation (3) has the form

$$
i \partial \psi(x, z) / d z=\hat{H}(z) \psi(x, z)
$$

where $\hat{H}(z)$ is the differential operator (parametrically dependent on $z) \hat{H}(z) \equiv \hat{T}+V(x, z)$. Here

$$
\hat{T} \equiv \frac{-1}{2 \beta_{0}} \frac{\partial^{2}}{\partial x^{2}} ; V(x, z) \equiv-k \Delta n(x, z) .
$$

This notation is in direct analogy to that used to describe the evolution of the quantum mechanical wavefunction $\psi$ for a one dimensional particle moving along spatial coordinate $x$ as a function of time (which becomes $z$ in the paraxial equation isomorphism). In the Schrödinger Equation [15], $\hat{T}$ is the particle's kinetic energy and $V$ is the potential energy which depends on $x$ (the particle's location) and time (or the location along the propagation axis, $z$, in the paraxial equation).

As noted above, we consider a class of guiding potentials $V(x, z)$ given by

$$
V(x, z)= \begin{cases}-V_{0} & \left|x-x_{c}(z)\right| \leq d \\ 0, & \left|x-x_{c}(z)\right|>d\end{cases}
$$

where the depth of the confining well is $V_{0}=k\left(n_{\text {guide }}-n_{0}\right)$. The function $x_{c}(z)$ specifies the center of the well in the $x$ direction at each point along the $z$ axis. Our goal is to optimize $x_{c}(z)$ in order to provide the most desirable wavepacket propagation, or in waveguide language, electric field profile. What constitutes a "good result"? Clearly, one criterion is that the output field, i.e., $\psi(x, L)$, resembles a prescribed target field $\psi_{\operatorname{Tar}}(x)$. The latter, for example, may correspond to the fundamental guided mode of a horizontal waveguide (oriented parallel to the $z$ axis) centered at $x_{f}$ and having width $2 d$. Thus, we wish to maximize $\left|\left\langle\psi_{\text {Tar }} \mid \psi(L)\right\rangle\right|^{2}$. Equivalently, we can define a "cost functional" $J=-\left|\left\langle\psi_{\text {Tar }} \mid \psi(L)\right\rangle\right|^{2}$, and seek the waveguide which minimizes this cost functional.

It may be desirable to take into account other features besides the quality of the output state. For example, for the S-bend waveguides considered here, the shorter the propagation distance over which the bend can be achieved, the more compact the device. This may be an important factor, particularly if the waveguide is to be used as an element of an optical circuit, where compactness is a virtue. One way to bias the optimization procedure in favor of rapidly bending waveguides is to add to the cost functional a term like $-\int_{0}^{L} d z\left[x_{c}(z)-x_{0}\right]^{2}$, i.e. a simple functional of the guiding trajectory $x_{0}(z)$ which penalizes structures that remain close to $x=x_{0}$ over a long propagation distance ("bend slowly"). Thus the specific cost functional we will utilize in this work is

$$
\begin{aligned}
J\left[x_{c}(z)\right]= & -\left|\left\langle\psi_{\operatorname{Tar}} \mid \psi(L)\right\rangle\right|^{2} \\
& -w \int_{0}^{L} d z\left[x_{c}(z)-x_{0}\right]^{2} .
\end{aligned}
$$

The parameter $w$ weights the relative importance of the two contributions to the cost. When $w=0$, only the quality of the output state (measured by its overlap with a prescribed target state) matters. As $w$ becomes increasingly positive, paths which "move away quickly" from $x=x_{0}$ become strongly favored, even though this entails some degradation of the output state. It is up to the designer to choose a value of $w$ which achieves the desired balance between these two factors.

There are many ways to parameterize the guiding path $x_{c}(z)$. A simple yet flexible representation can be given in terms of a Fourier series. Specifically

$$
x_{c}(z)=x_{0}+\left(x_{f}-x_{0}\right) z / L+\sum_{k=1}^{N} a_{k} \sin (k \pi z / L)
$$

where the first term on the RHS provides a straight-line path between $x_{0}$ and $x_{f}$ over a propagation distance $L$, while the series of sine waves enables representation of a wide variety of paths between these fixed endpoints. (As $N \rightarrow \infty$, arbitrary paths can be constructed.) Thus, our task is to pick the set of Fourier coefficients $a_{1}-a_{N}$ corresponding to the index profile that guides the incident lightwave in an optimal manner, i.e., has the lowest cost $J$ associated with it.

When the trial path contains only one or two Fourier components, direct enumeration can be used to search all possibilities. However, the number of configurations of $\vec{a}=$ $\left(a_{1}, a_{2}, \cdots, a_{N}\right)$ which must be considered goes up geometrically with $N$, so this "brute force" procedure will not be useful in the general case. Fortunately, optimal control theory [14] provides an efficient recipe for computing the gradient of $J(\vec{a})$ at a particular configuration. Given $\vec{\nabla} J$ we can then use a simple annealing procedure like steepest descents [17] to quickly locate a minimum of the function $J(\vec{a})$. The Fourier coefficients for which $J$ is a minimum are at least locally optimal, i.e., provide the best guiding of any set near the initially guessed values. More advanced minimization techniques such as simulated annealing [17] may be utilized to check for the presence of other, deeper lying minima, in the $J(\vec{a})$ landscape, if these are suspected to exist.

The recipe for $\vec{\nabla} J(\vec{a})$ is obtained by the method of Lagrange multipliers [16]. That is, the cost function $J$ is modified to

$$
\begin{aligned}
J(\vec{a})= & -\langle\psi(L)|\hat{O}| \psi(L)\rangle-w \int_{0}^{L} d z\left(x_{c}(z)-x_{0}\right)^{2} \\
& -\int_{0}^{L} d z\left\{\left\langle\lambda(z)\left|\left[i \frac{\partial}{\partial z}-\hat{H}(z)\right]\right| \psi(z)\right\rangle+\text { c.c. }\right\} .
\end{aligned}
$$


The modification of the first term on the RHS is cosmetic. The projection operator $\hat{O} \equiv\left|\psi_{\text {Tar }}\right\rangle\left\langle\psi_{\text {Tar }}\right|$ provides another way to express the condition $\langle\psi(L)|\hat{O}| \psi(L)\rangle=$ $\left|\left\langle\psi_{\operatorname{Tar}} \mid \psi(x, L)\right\rangle\right|^{2}$. (Introduction of this projection operator simplifies the derivation of the expression for $\vec{\nabla} J$; cf., the Appendix.)

The final term (2nd line) on the r.h.s. of (9) is a Lagrange multiplier addition which expresses the condition that only $\psi(x, z)$ which satisfy the paraxial equation are considered in calculating the "cost" associated with a particular Fourier coefficient vector $\vec{a}$. (Here and below c.c. indicates "complex conjugate.") The auxiliary function $\lambda(x, z)$ is a set of Lagrange multipliers [one for each $(x, z)$ ]. It is determined by considering unconstrained variations of the $a_{k}$ and using the constraint that $\psi$ must satisfy the paraxial equation at an appropriate stage of the derivation.

Following Rabitz and coworkers [14] (some details are given in the Appendix), one finds that each gradient component can be calculated as

$$
\begin{aligned}
\frac{\partial J}{\partial a_{k}}= & 2 \operatorname{Re} \int_{0}^{L} d z\left\langle\lambda(z)\left|\frac{\partial V}{\partial a_{k}}\right| \psi(z)\right\rangle \\
& -w \int_{0}^{L} d z \frac{\partial}{\partial a_{k}}\left[x_{c}(z)-x_{0}\right]^{2} .
\end{aligned}
$$

Once we have arrived at this expression, the practical procedure consists of a sequence of steps. First, $|\psi(z)\rangle$ is propagated forward from $|\psi(0)\rangle$ under the Hamiltonian $H(t)=\hat{T}+$ $V(x, z)$, with $\hat{T}$ the 1-d kinetic energy operator and $V(x, z)$ the effective "guiding" potential indicated in (5). Second, the auxiliary wavefunction $\lambda(x, z)$ is obtained by propagating a function which essentially corresponds to the target state $\psi_{\operatorname{Tar}}(x)$ backward from $z=L$ to $z=0$ along the same potential. The precise description of $\lambda(x, z)$ at the boundary $z=L$ is

$$
|\lambda(L)\rangle=-i \hat{O}|\psi(L)\rangle=-i\left\langle\psi_{\operatorname{Tar}} \mid \psi(L)\right\rangle\left|\psi_{\operatorname{Tar}}\right\rangle
$$

and the equation of motion for evolving $\lambda(x, z)$ backward along the same guiding potential (index of refraction profile) to $z=0$ is

$$
i \frac{\partial}{\partial z}|\lambda(z)\rangle=\hat{H}(z)|\lambda(z)\rangle .
$$

Third, the different gradient components are computed using (10). The derivation of these results is presented in the Appendix. Finally, the technique of steepest descents can be used to "march downhill" until a minimum in the function $J$ is reached. In this method [17], the Fourier coefficients are updated according to $a_{k} \rightarrow a_{k}-\epsilon \partial J / \partial a_{k}$, where $\epsilon$ is a small positive number (an infinitesimal time step). For small enough $\epsilon$ the method is guaranteed to find a minimum value of the function $J\left(a_{1}, \cdots, a_{N}\right)$, which corresponds to an (at least locally) optimal waveguide design. In the next section we illustrate the method for prototypical S-bend waveguides.

\section{NUMERICAL ILLUSTRATIONS}

Fig. 1 shows typical S-bend guides, whose purpose is to move light injected at lateral position $x=x_{0}$ to a different

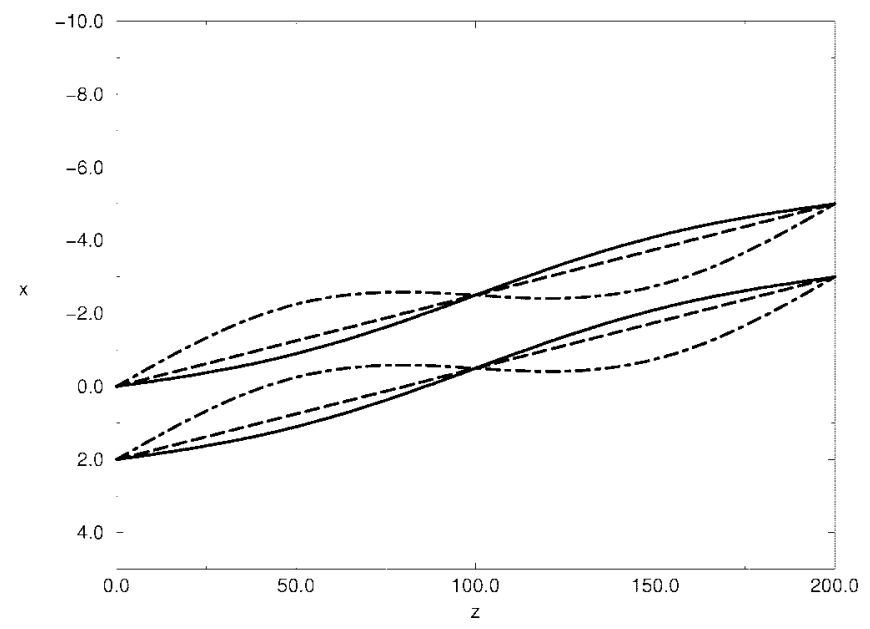

Fig. 1. S-bend waveguides with center paramaterized by one Fourier coefficient, $a_{2}$. Solid, dashed, and dot-dashed show cases $a_{2}=0.35,0.0,-1.0$, respectively. (All distances are in microns.)

lateral position $x=x_{f}$ at the end of the waveguide. Here, we choose $x_{0}=1 \mu, x_{f}=-4 \mu$. As noted above, in the interior of the guide the refractive index is $n_{\text {guide }}$ and outside the guide the refractive index is $n_{0}$. The numerical values utilized in the calculations presented in this section are $n_{\text {guide }}=3.568$ and $n_{0}=3.558$. (The parameters specified in this paragraph are typical for a variety of waveguides characterized from GaAs/AlGaAs composites [5]-[7].) For each value of $z$, the index profile along the $x$ direction is the same except for the shift in the center of the guiding region. The specific form of the lateral variation of the guide is as given in (6), with the numerical value $d=1 \mu$ (i.e., the width of the guide is $2 \mu$ ). The length of the guide in the propagation direction is $L=200 \mu$ and the free-space wavelength of the propagated light is $0.87 \mu$.

The center of the guide varies with $z$ according to the function $x_{c}(z)$. This function can be parameterized as a straight line connecting the endpoints $x_{0}$ and $x_{f}$ plus a superposition of sine waves that vanish at the endpoints. By varying the coefficients of the sine waves a variety of curves can be represented. To illustrate, we begin with the case of one sine wave, i.e.

$$
x_{c}(z)=x_{0}+\left(x_{f}-x_{0}\right) z / L+a_{2} \sin (2 \pi z / L) .
$$

The value $a_{2}=0$ obviously corresponds to a straight line connecting path (dashed lines in Fig. 1). Increasing $a_{2}$ introduces curvature into the guiding path. With the class of guiding paths parameterized by (13), $J$ is a function of only one variable $a_{2}$. Hence, we can determine the function $J\left(a_{2}\right)$ by propagating $\psi(x, z)$ under the paraxial equation for the index profile determined by each value of $a_{2}$, and then evaluating the RHS of (7).

More specifically, given an initial electric field amplitude distribution $\psi(x, 0)$ and an index of refraction profile, the paraxial equation (4) provides unique values for the amplitude at all distances along the propagation axis, i.e., $\psi(x, z)$. There are a number of reliable ways to propagate the amplitude to $z>0$ on a spatial grid. One of the most efficient, accurate, and 


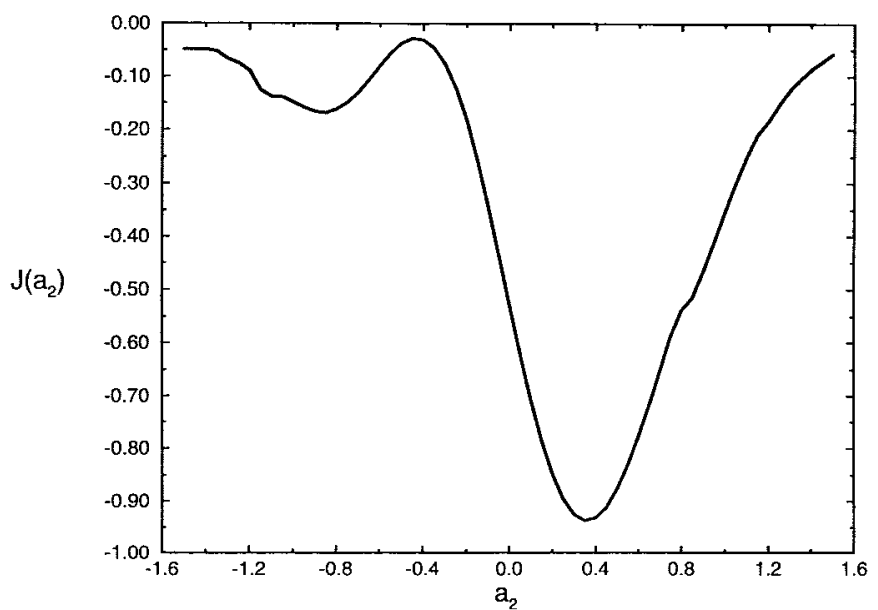

Fig. 2. Plot of cost function $J$ versus $a_{2}$ for waveguide center parameterized by one Fourier coefficient (cf. Fig. 1), and $w=0$ (only wavepacket overlap is considered).

flexible is the split operator method (SOM) of Feit and Fleck, ${ }^{1}$ which has been utilized in all calculations presented here.

Given $\psi(x, L)$ [output from the split operator amplitude propagation calculation], (7) calls for overlap with a target field amplitude $\psi_{\operatorname{Tar}}(x)$. The natural choice for $\psi_{\operatorname{Tar}}$ in the present illustration is the fundamental guided mode of the "straight guide" (extending along the $z$-axis without curvature) that connects to the endpoint of our S-guide at $z=L$. The index profile for this guide is specified by (6) with $x_{c}(z) \rightarrow x_{c}(L)$. The guide's fundamental mode is the eigenfunction of the operator $\hat{H}(L)$ which corresponds to the smallest eigenvalue. We compute this eigenfunction in the standard manner (by matching $\psi$ and $d \psi / d x$ at the $x$-values where the index function is discontinuous [1]). The overlap with $\psi(x, L)$ is then performed via numerical quadrature.

The result when $w=0$ is shown in Fig. 2. As can be seen, the global minimum in $J$ occurs for $a=0.35$ and corresponds to an output state characterized by $\left|\left\langle\psi_{\operatorname{Tar}} \mid \psi(L)\right\rangle\right|^{2}=0.95$. This guiding is considerably better than for a straight line path $a_{2}=0$, when $\left|\left\langle\psi_{\operatorname{Tar}} \mid \psi(L)\right\rangle\right|^{2}=0.55$. So the strategy of locating the global minimum of $J$ is evidently prudent.

Most importantly, we have checked that the Optimal Control procedure generates the slope $\partial J / \partial a_{2}$ reliably at each value of $a_{2}$. Recall that this entails forward propagation of $\psi(x, z)$, then backward propagation of an auxiliary wavepacket $\lambda(x, z)$ [cf., (12)]. We have further checked that this procedure for derivative evaluation can be combined with a steepest descents algorithm based on these derivatives in order to locate the minimum in $J$. Provided that we make a decent initial guess, e.g., $a_{2}=0$, the algorithm, which we shall term the "Optimal Control/Steepest Descent (OC/SD) protocol," finds the global minimum with no problem. The topography of $J\left(a_{2}\right)$ in Fig. 2 illustrates the generic feature that $J$ is a complicated function of its arguments and will in general have more than one minimum. In the present case, it would require a poor guess (say, $a_{2} \approx-1$, which produces a bizarre S-bend guide; for example, the dot-dashed in Fig. 1) to get stuck in

\footnotetext{
${ }^{1}$ Back-propagation along the $z$-axis was also performed using the Split Operator method [9].
}

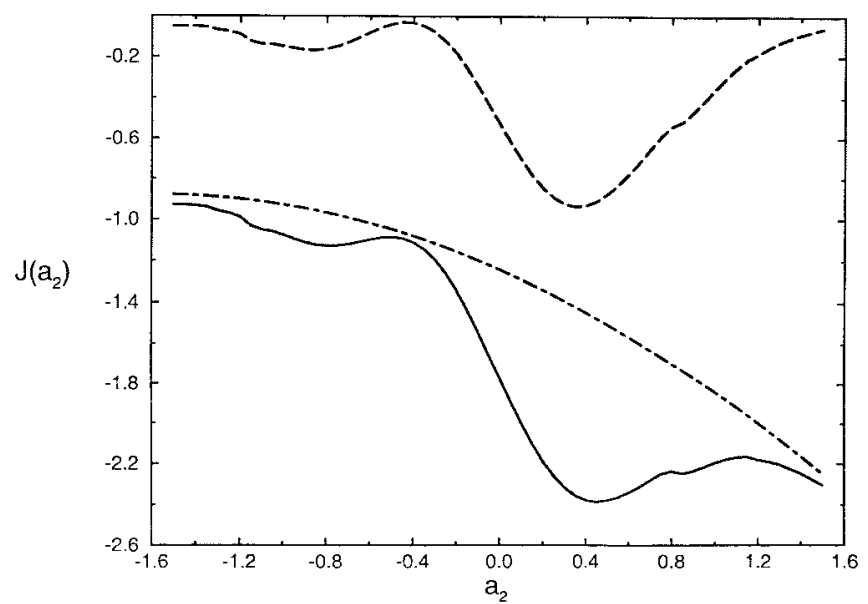

Fig. 3. Solid line shows cost function $J$ versus $a_{2}$ for waveguide center parameterized by one Fourier coefficient (cf., Fig. 1), and $w=0.0015$ (weighting factor for rapid bend is included). This curve is decomposed into the wavepacket overlap term (same as in Fig. 2), shown via dashed line, and the rapid bend weight term, shown via dash-dotted line.

a local minimum that is far from the global minimum. In some problems it may be necessary to couple the steepest descents strategy with a method like simulated annealing [17] or Brownian dynamics [18] to prevent trapping in local minima. Fortunately, in many cases, like the ones presented here and below, a good intuitive guess is possible. The OC/SD protocol then quickly improves upon the initial guess.

Now we consider optimization subject to the additional condition that the guide bends as rapidly as possible. This is realized by the "penalty" functional proportional to parameter $w$ in (7). We show as the solid line in Fig. 3 modification of $J$ for the value of $w=0.0015$. This curve is decomposed into its two components, namely the "overlap quality" term (dashed line, same data as in Fig. 2), and the "penalty term" [dash-dotted line, representing the second term in (7)]. Note that the addition of the penalty term results in a shift in the minimum of $J$, which now occurs at $a_{2}=0.47$. This shift incorporates the desired increase in the rapidity of the bend in the waveguide (slightly beyond that of the case $a_{2}=0.35$ shown in Fig. 1). Also note that the output quality associated with the "optimized waveguide" is approximately 0.90, i.e., somewhat reduced from that obtained when $w=0$. However, this is still much better than the output quality associated with the straight line waveguide discussed above. The compromise between the two terms in the cost functional is "designer controlled" by the choice of $w$.

To demonstrate the utility of the optimal control method we next consider a guiding curve represented by two Fourier coefficients, i.e.

$$
\begin{aligned}
x_{c}(z)= & x_{0}+\left(x_{f}-x_{0}\right) z / L+a_{2} \sin (2 \pi z / L) \\
& +a_{4} \sin (4 \pi z / L)
\end{aligned}
$$

A contour plot of $J$ vs $a_{2}, a_{4}$ is shown in Fig. 4 for the case $w=0$ (overlap optimization only). It is apparent that the minimizing Fourier configuration is approximately $\left(a_{2}, a_{4}\right)=(0.33,-0.034)$. Fig. 5 shows the corresponding plot when $w=0.0015$. In this case, the global minimum 


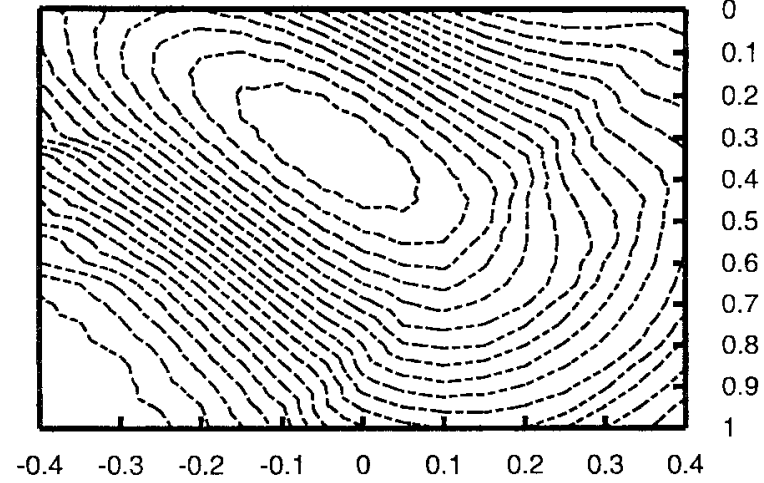

$a_{4}$

Fig. 4. Contour plot of $J$ versus $a_{2}, a_{4}$ for case where waveguide center is parameterized by these two Fourier coefficients, and $w=0$ (only wavepacket overlap is considered). Note the (global) minimum at $\left(a_{2}, a_{4}\right) \cong(0.33,-0.034)$.

occurs at approximately $\left(a_{2}, a_{4}\right)=(0.6,0.1)$. These plots were obtained by a direct scan of the $\left(a_{2}, a_{4}\right)$ configuration space, propagating $\psi(x, z)$ for each $\left(a_{2}, a_{4}\right)$ point on a square grid. The scaling of numerical effort to carry out this procedure grows geometrically with number of Fourier coefficients (hence the relatively coarse grid spacing utilized in these figures). The scaling of the OC/SD strategy is much better. The only real issue is whether (10) produces an accurate value of the gradient of $J$ for any $\left(a_{2}, a_{4}\right)$. We have checked that it does. Given this fact, the computational effort associated with the procedure is guaranteed to scale mildly with number of Fourier coefficients. Evaluation of the gradient (in all Fourier coefficient directions) requires only one forward and one backward wavepacket propagation according to the paraxial equation. The extraction of all components of the gradient is then nearly instantaneous. ${ }^{2}$ Finally, the steepest descent procedure exhibits a roughly linear scaling with number of Fourier coefficients (the number of "sweeps" needed is nearly independent of dimension of the configuration space). We have checked that, starting from the initial guess $\left(a_{2}, a_{4}\right)=(0,0)$, the OC/SD protocol easily locates the global minima indicated in Figs. 4 and 5.

It is instructive to plot the electric field distribution $\psi$ associated with the "optimized" waveguide structure. The waveguide which produces the best output state [i.e., the one with $\left.\left(a_{2}, a_{4}\right)=(0.33,-0.034)\right]$ is shown in Fig. 6 , along with the associated electric field distribution. The output state is compared visually to the target state (and the initial state) in Fig. 7. The overlap measure has the value $\left|\left\langle\psi_{\operatorname{Tar}} \mid \psi(L)\right\rangle\right|^{2}=$ 0.94 for this waveguide. This should be contrasted with the

${ }^{2}$ It is easy to see that for the form of $x_{c}(z)$ utilized in our illustration [(8)], the second integral in (10) has the structure $\int_{0}^{L} d z g(z) \sin (k \pi z / L)$, where $g(z)$ is a function which is independent of the particular value of $a_{k}$ for which $\partial J / \partial a_{k}$ is being computed. In fact, the first integral in (10) has the same structure. For this integral, the function $g(z)$ depends on the details of the wavepackets $|\psi(z)\rangle$ and $|\lambda(z)\rangle$. But, again, it is the same function for all $a_{k}$. Thus, evaluation of $\partial J / \partial a_{k}$ is achieved via a one dimensional quadrature, which can be done "instantaneously" on current computer workstations. Furthermore, the work required to calculate $\vec{\nabla} J\left(\partial J / d a_{k}\right.$ for all Fourier coefficients) scales linearly with the number of coefficients included in the series.

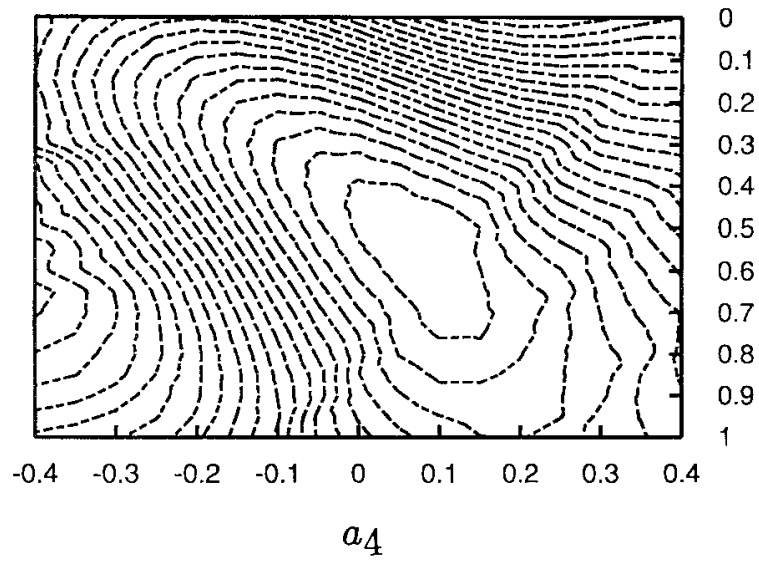

$a_{2}$

Fig. 5. Contour plot of $J$ versus $a_{2}, a_{4}$ for case where waveguide center is parameterized by these two Fourier coefficients, and $w=0.0015$ (weighting factor for rapid bend is included). Note the (global) minimum at $\left(a_{2}, a_{4}\right) \cong(0.6,0.1)$.

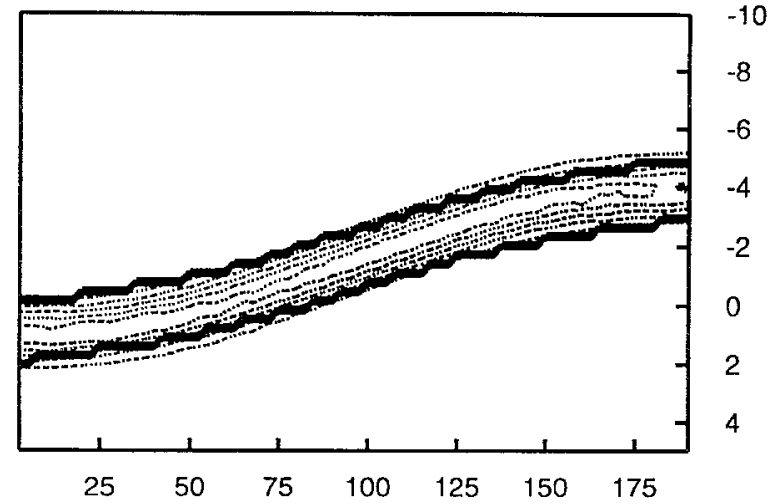

Fig. 6. S-bend waveguide (edges shown via solid lines) and the associated electric field intensity $|\psi(x, z)|^{2}$ (shown via dashed line) for the optimized, $w=0$ guide parameterized by two Fourier coefficients, namely $\left(a_{2}, a_{4}\right) \cong(0.33,-0.034)$. (All distances in this and subsequent figures are in microns. Also note that the "staircase" features in the edge demarcation lines are artifacts of the contour plotting program. The actual waveguide edges are smooth, as depicted in Fig. 1.)

corresponding straight waveguide results, $\left(a_{2}, a_{4}\right)=(0,0)$, which are shown in Figs. 8 and 9. The overlap is 0.52 for this unoptimized structure. The structure generated with the optimization procedure in the case that $w=0.0015$ is shown in Fig. 10, with the associated output state depicted in Fig. 11. Here the overlap is 0.82 , which is somewhat lower than the maximum value obtainable (though still considerably better than that obtained from the straight structure.) However, this waveguide bends more rapidly than either of the other two structures, and hence delivers the light to the target position over a shorter distance, as desired.

\section{CONCLUSIONS}

In this paper we have adapted the principles of optimal control theory, which have been developed over the last decade by the quantum dynamics community [14] to improve control of laser-molecule interactions, to the problem of designing optical waveguides. Our task was made easy by the fact that electric fields in such waveguides are well described by the paraxial equation of scalar optics, and the paraxial 


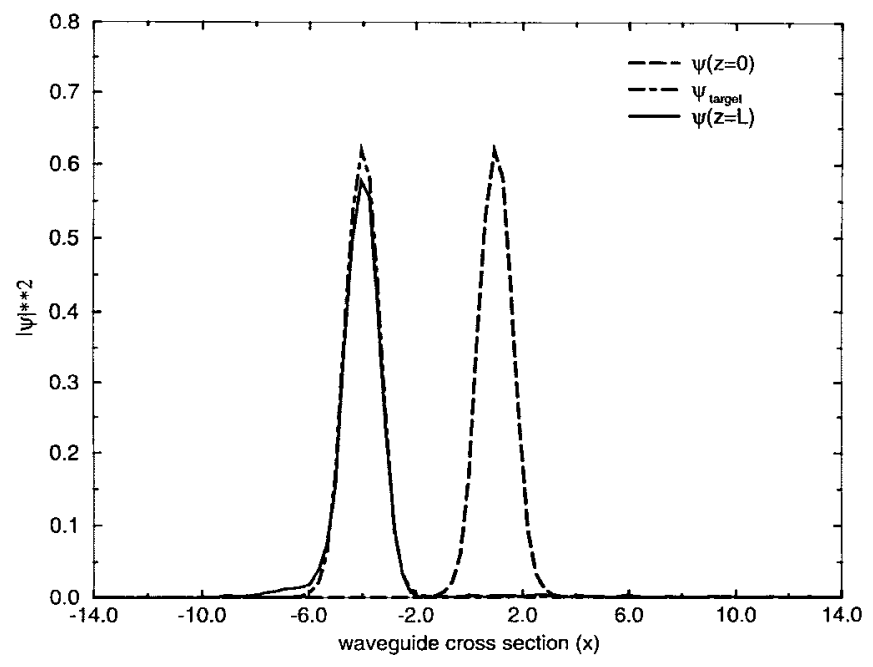

Fig. 7. Output electric field intensity $|\psi(x, z)|^{2}$ associated with guide depicted in Fig. 6 is shown via solid line. Target field intensity is shown via dot-dashed line; lateral intensity distribution of field incident at $z=0$ is shown via dashed line.

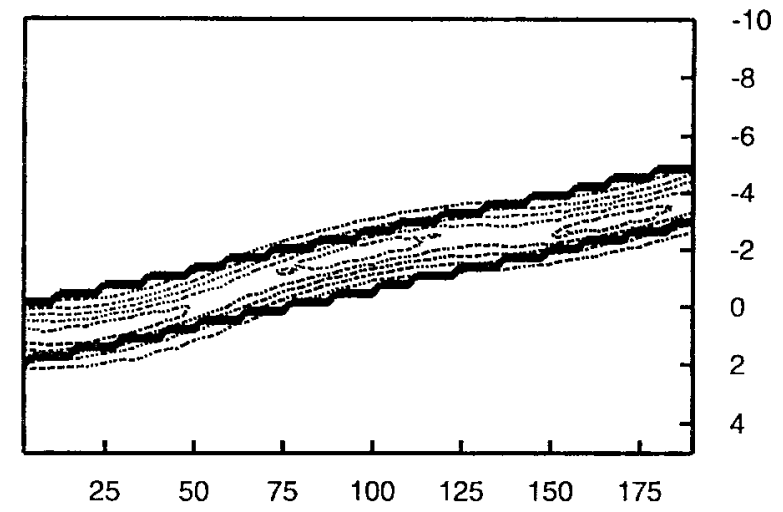

Fig. 8. S-bend waveguide (edges shown via solid lines) and the associated electric field intensity $|\psi(x, z)|^{2}$ (shown via dashed line) for straight waveguide.

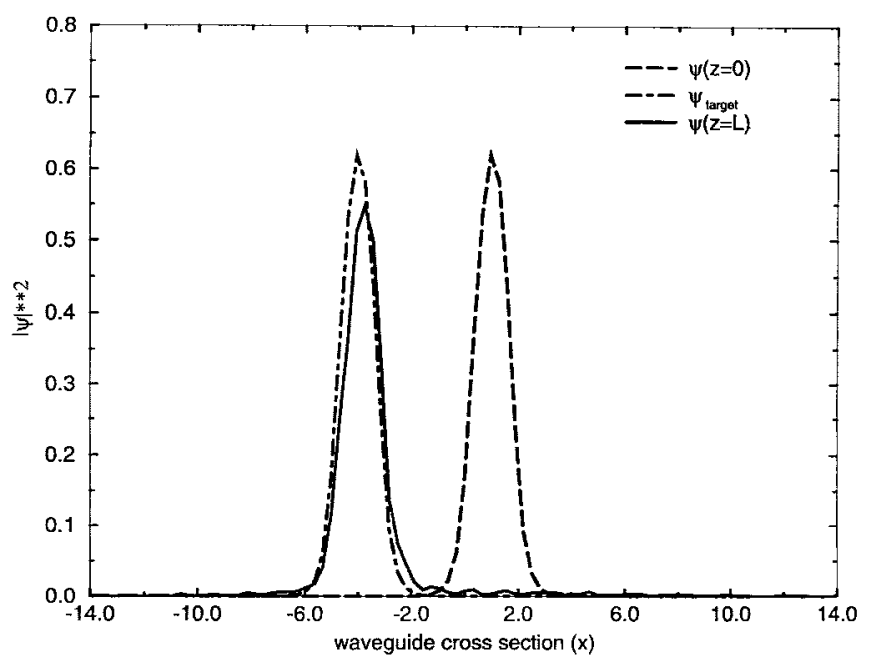

Fig. 9. Output electric field intensity $|\psi(x, z)|^{2}$ associated with straight waveguide depicted in Fig. 8 is shown via solid line. Target field intensity is shown via dot-dashed line; lateral intensity distribution of field incident at $z=0$ is shown via dashed line.

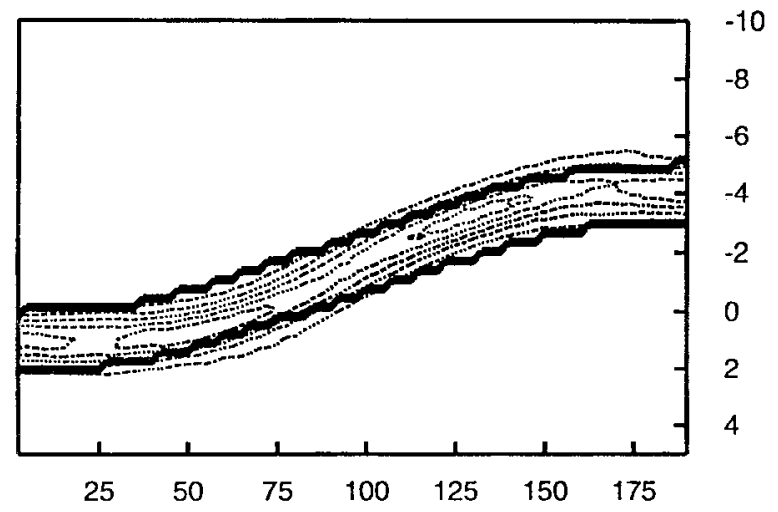

Fig. 10. S-bend waveguide (edges shown via solid lines) and the associated electric field intensity $|\psi(x, z)|^{2}$ (shown via dashed line) for the optimized, $w=0.0015$ guide parameterized by two Fourier coefficients, namely $\left(a_{2}, a_{4}\right) \cong(0.6,-0.1)$.

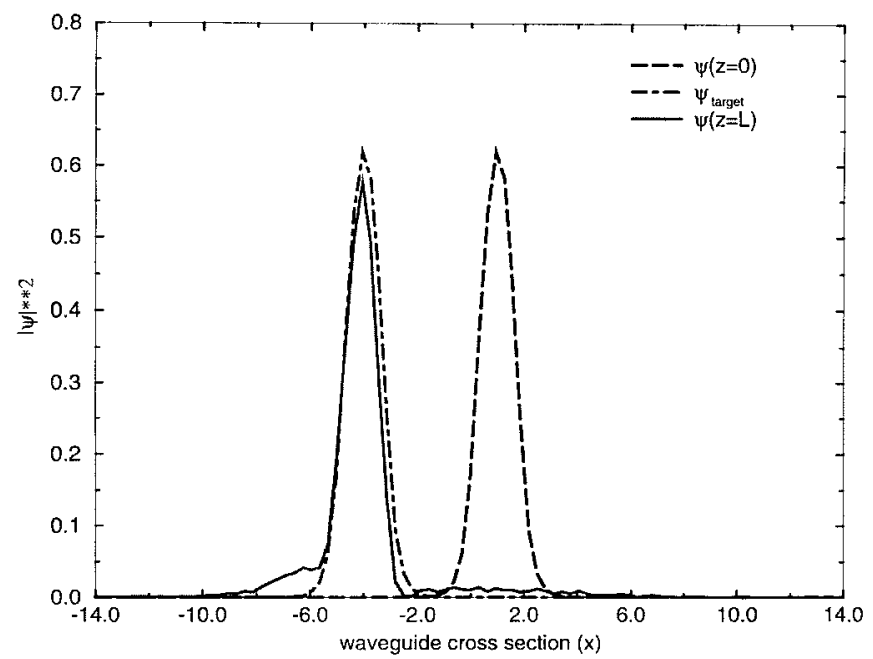

Fig. 11. Output electric field intensity $|\psi(x, z)|^{2}$ associated with guide depicted in Fig. 10 is shown via solid line. Target field intensity is shown via dot-dashed line; lateral intensity distribution of field incident at $z=0$ is shown via dashed line.

equation is a time-dependent Schrödinger Equation of the type used in quantum dynamics. Of course, the details of the "time-dependent potential energy function" associated with the waveguide design problem are different here, as are the associated optimal design features (e.g., additional goals besides producing a desired output electric field).

Optimal control theory begins with a cost function, which indicates how well a particular waveguide performs. (The waveguide is specified in terms of some number of variable parameters. In the illustration presented here, Fourier coefficients were used to define the guiding path of an S-bend waveguide.) It then prescribes an efficient way to compute the gradient of the cost function for any set of parameters defining the waveguide structure. Knowledge of the gradient can be coupled with simple multidimensional minimization techniques like steepest descents to find the parameter set that gives the minimum (optimal) value of the cost function.

We demonstrated the basic principle of the optimal control/steepest descents protocol with some illustrative examples. The encouraging scaling of effort with the number of variable 
parameters considered in the waveguide design was clearly indicated. We note that the method is in no way restricted to a Fourier series description of the guiding center of a single waveguiding structure. A wide variety of variations can be considered, including changes in the width of the guide in certain sections (as exploited, for example, in multimode interference [MMI] devices [19]), or the index of the guiding region as a function of position along the propagation direction [20]. Compound waveguide structures such as Y-junctions [21] and Mach-Zehnder interferometers [22] can also be analyzed. We hope that the availability of an efficient, automated procedure for optimizing waveguide structures will lead to improvements in their functionality (e.g., in optoelectronic applications).

\section{APPENDIX}

Consider the change in $J$ made by a small change of Fourier coefficients $\left\{\delta a_{k}\right\}$ around their current configuration $\left\{a_{k}\right\}$. Clearly, on the left hand-side (LHS) of (9), $J \rightarrow$ $J+\sum_{k=1}^{N}\left(\partial J / \partial a_{k}\right) \delta a_{k}$. To calculate the first order variation of $J$ on the RHS, we must consider variations induced on the Hamiltonian (potential function), on $x_{c}(z)$, and on the propagated wavefunction $\psi(x, z)$ and the (still to be determined) Lagrange multipliers $\lambda(x, z)$. That is, we substitute $a_{k} \rightarrow a_{k}+\delta a_{k},|\psi(z)\rangle \rightarrow|\psi(z)\rangle+|\delta \psi(z)\rangle$, and $|\lambda(z)\rangle \rightarrow$ $|\lambda(z)\rangle+|\delta \lambda(z)\rangle$. Following standard procedure in the Calculus of Variations [23], only terms which entail no variation or are first order in one of the variations are retained. This leads to an overall first order variation $\delta J$ which can be broken into five terms ${ }^{3}$

$$
\delta J=-([\mathrm{I}]+[\mathrm{II}]+[\mathrm{III}]+[\mathrm{IV}]+[\mathrm{V}]) .
$$

The terms are

$$
\begin{aligned}
{[\mathrm{I}] } & =\langle\delta \psi(L)|\hat{O}| \psi(L)\rangle+\text { c.c. } \\
{[\mathrm{II}] } & =w \sum_{k=1}^{N} \delta a_{k} \int_{0}^{L} d z \frac{\partial}{\partial a_{k}}\left[x_{c}(z)-x_{0}\right]^{2} \\
{[\mathrm{III}] } & =\int_{0}^{L} d z\left[\left\langle\lambda(z)\left|\left(i \frac{\partial}{\partial z}-\hat{H}(z)\right)\right| \delta \psi(z)\right\rangle+\text { c.c. }\right] \\
{[\mathrm{IV}] } & =\int_{0}^{L} d z\left[\left\langle\delta \lambda(z)\left|\left(i \frac{\partial}{\partial z}-\hat{H}(z)\right)\right| \psi(z)\right\rangle+\text { c.c. }\right] \\
{[\mathrm{V}] } & =-\sum_{k=1}^{N} \delta a_{k} 2 \operatorname{Re} \int_{0}^{L} d z\left\langle\lambda(z)\left|\frac{\partial \hat{H}}{\partial a_{k}}\right| \psi(z)\right\rangle .
\end{aligned}
$$

Of these terms, [I] is generated by considering variations in the first (target overlap) term on the RHS of (9), [II] comes from varying the second ("design goal") term, and [III, IV, V] from the third (Lagrange multiplier) terms.

First note that [IV] vanishes, since the wavepacket $\psi(x, z)$ associated with the current set of Fourier coefficients satisfies the Schrödinger Equation associated with the same coefficients. Furthermore, by choosing the Lagrange multipliers $\lambda(x, z)$ appropriately, we can arrange for the sum $[\mathrm{I}]+[\mathrm{III}]=0$.

\footnotetext{
${ }^{3}$ The boundary condition $\mid \delta \psi(0)=0$, which reflects the fact that the initial value of the amplitude function $\psi(x, 0)$ is fixed, has also been employed to obtain this formula.
}

This requires that $\lambda(x, z)$ satisfy

$$
i \frac{\partial}{\partial z}|\lambda(z)\rangle=\hat{H}(z)|\lambda(z)\rangle
$$

subject to the boundary condition that

$$
|\lambda(L)\rangle=-i \hat{O}|\psi(L)\rangle
$$

To summarize the content of (16) and (17). Take the output wavepacket $\psi(x, L)$ and form the wavepacket $\lambda(x, L)$ as indicated in (17). (Note: For the choice of $\hat{O}$ adopted here, $\lambda(x, L)$ is equal to $\psi_{\operatorname{Tar}}(x)$ to within an overall constant.) Then, backpropagate $\lambda(x, L)$ to $z=0$. As noted above, this choice of $\lambda(x, z)$ results in the condition $[\mathrm{I}]+[\mathrm{III}]=0$. By matching the remaining terms on the r.h.s. of (9) with those on the left, the identification of $\partial J / \partial a_{k}$ noted in (10) is achieved.

\section{REFERENCES}

[1] D. Marcuse, Theory of Dielectric Waveguides, 2nd ed. New York: Adademic, 1991

[2] J. D. Jackson, Classical Electrodynamics, 2nd ed. New York: Wiley, 1975.

[3] K. S. Yee, "Numerical solution of intitial value problems involving Maxwell's equations in isotropic media," IEEE Trans. Antennas Propagat., vol. AP-14, p. 302, 1966.

[4] A. Taflove and M. E. Brodwin, "Numerical solution of steady-state electromagnetic scattering problems using the time-dependent Maxwell's equations," IEEE Trans. Microwave Theory Tech., vol. MTT-23, p. 623, 1975.

[5] D. W. Langer, M. Chen, H. Lee, and M. Chemielowski, "Quantum well structures for integrated opto-electronics," in Proc. SPIE Digital Opt. Comput. II, 1990, vol. 1215, pp. 243-241.

[6] R. D. Coalson, D. K. Pant, A. Ali, and D. W. Langer, "Computing the eigenmodes of lossy field-induced optical waveguides," J. Lightwave Technol., vol 12, pp. 1015-1022, 1994.

[7] D.-S. Min, D. W. Langer, D. K. Pant, and R. D. Coalson, "Design and analysis of wide-angle Y-branch waveguide with low-losses for integrated optics," SPIE Proc., vol. 3006, pp. 459-467, 1997; see also, D.-S. Min, D. W. Langer, D. K. Pant, and R. D. Coalson, "Wide-angle low-loss waveguide branching for integrated optics," Fiber Integrated Opt., vol 16, pp. 331-342, 1997.

[8] A. H. Cherin, An Introduction to Optical Fibers. New York: McGrawHill, 1983.

[9] M. D. Feit, J. A. Fleck, Jr., and A. Steiger, "Computation of mode properties in optical fiber waveguides by a propagating beam method,"Appl. Opt., vol. 19, pp. 1154-1164, 1980.

[10] I. Vorobeichik, U. Peskin, and N. Moiseyev, "Modal losses and design of modal irradiance patterns in an optical fiber by the complex scaled $\left(t, t^{\prime}\right)$ method," J. Opt. Soc. Amer. B, pp. 1133-1141, 1995.

[11] R. Kosloff, "Time-dependent quantum-mechanical methods for molecular dynamics," J. Phys. Chem., vol. 92, pp. 2087-2100, 1988.

[12] A. H. Zewail, "Femtochemistry-Advances over a decade," in Femtochemistry: Ultrafast Chemical and Physical Processes in Molecular Systems, M. Chergui, Ed. Singapore: World Scientific, 1996, pp. 3-14.

[13] B. Kohler, V. Yakovlev, J. Che, J. L. Krause, M. Messina, K. R. Wilson, N. Schwentner, R. M. Whitnell, and Y. Yan, "Quantum control of wave packet evolution with tailored femptosecond pulses," Phys. Rev. Lett., vol. 74, p. 3360, 1995.

[14] S. Shi and H. Rabitz, J. Chem. Phys., vol. 92, p. 364, 1990; see also, H. Rabitz, "Adaptive feedback control of molecular motion" in Frontiers of Chemical Dynamics," E. Yurtsever, Ed., p. 181, NATO ASI Series, Kluwer (1995); see also, "Optimally designed potentials for control of electron-wave scattering in semiconductor nanodevices;" see also, P. Gross, V. Ramakrisna, E. Vilallonga, H. Rabitz, M. Littman, S. A. Lyon, and M. Shayegan, Phys. Rev. B, vol. 49, pp. 11100-11110 (1994); see also, D. Neuhauser and H. Rabitz, "Paradigms and algorithms for controlling molecular motion," Accts. Chem. Res., vol. 26, pp. 496-501, 1993. 
[15] D. A. McQuarrie, Quantum Chemistry Mill Valley, CA: University Science Books, 1983.

[16] T. A. Apostol, Calculus, v. 2, 2nd ed. Waltham, MA: Xerox College Publishing, 1969), Section 9.14.

[17] W. H. Press, B. P. Flannery, S. A. Teukolsky, and W. T. Vetterling, Numerical Recipes (Cambridge Univ. Press, Cambridge, 1998), Chapt. 10.

[18] J. D. Madura, M. E. Davis, M. K. Gilson, R. C. Wade, B. A. Luty, and J. A. McCammon, "Biological applications of electrostatics calculations and Brownian dynamics simulations," Chapt. 4, Reviews in Computational Chemistry, vol. 5, pp. 229-267, 1994.

[19] C. Rolland, D. M. Adams, D. Yevick and B. Hermansson, "Optimization of strongly guiding semiconductor rib waveguide Y-junctions," IEEE Photon. Technol. Lett., vol. 2, p. 404, 1990.

[20] W. H. Hung, H. P. Chan, and S. P. Chung, "Novel design of wideangle single-mode symmetric Y-junctions," Electon. Lett., vol. 24, p. 1184, 1988.

[21] K. Tsutsumi, Y. Imada, H. Hirai, and Y. Yuba, J. Lightwave Technology, vol. 6, p. 590, 1988; see also, H. Hatami-Hanza, P. L. Chu, and M. J. Lederer, IEEE Photon. Technol. Lett., vol. 6, p. 528, 1994.

[22] J. E. Zucker, K. L. Jones, B. I. Miller, and U. Koren, IEEE Photon Technol. Lett., vol. 2, p. 32, 1990; see also, J. S. Cites and P. R. Ashley, J. Lightwave Technol., vol. 12, p. 1167, 1994.

[23] R. Weinstock, Calculus of Variations, with Applications to Physics and Engineering. New York: Dover, 1974.

D. K. Pant was born in Pune, India, in 1967. He received the Master's degree in chemistry from the Indian Institute of Technology (IIT), Kanpur, in 1990. $\mathrm{He}$ is currently working toward the Ph.D. degree in chemistry at the University of Pittsburgh, Pittsburgh, PA, on dielectric optical waveguide design.
Rob D. Coalson was born in Tucson, AZ, in 1955. He received the A.B. degree in physics and chemistry from Harvard College, Cambridge, MA, in 1977 and the Ph.D. degree in chemical physics from Harvard University, Cambridge, MA, in 1984

After a Postdoctoral appointment at Los Alamos National Laboratory, he joined the Univerity of Pittsburgh, PA, where he is now Professor of Chemistry and Professor of Physics. His research interests include the theory of manybody quantum dynamics, the structure and function of colloid crystals, and optical waveguide design.

Marta I. Hernández was born in Santa Cruz de Tenerife, Spain, in 1963. She received the degree in physics from Universidad Complutense de Madrid, Spain, in 1987 and the Ph.D degree in physics from Universidad de La Laguna, Spain, in 1992.

After a Postdoctoral appointment at the University of Cambridge, U.K., during 1993-1994, she joined the Institute of Mathematics and Fundamental Physics, (C.S.I.C.), Madrid, where she is currently a Research Assistant. Her research interests include the application of quantum dynamcis to reactive scattering and molecular clusters and, more recently, to waveguide design.

José Campos-Martínez was born in Madrid, Spain, in 1960. He received the degree in chemistry in 1982 from Universidad Complutense de Madrid, Spain, and the Ph.D. degree in chemistry from the same university.

He was a Fullbright Fellow at the University of Pittsburgh, Pittsburgh, PA (1989-1991), and he is currently working at the Institute of Mathematics and Fundamental Physics, (C.S.I.C.) in Madrid. His research interests include fundamental processes in molecular physics, and lately, optical waveguide design problems. 